\title{
Variability in Wild Pomegranate (Punica granatum l.) Population from Ganderbal and Budgam Districts of Kashmir Valley
}

\author{
Imtiyaz A. Wani*, M. Y. Bhat, Shaiq A. Ganai, Abid A. Lone, Aarifa Jan and M. M. Mir \\ Division of Fruit science SKUAST-K Shalimar, Srinagar-191121, India; imtiyazwani91@yahoo.in
}

\begin{abstract}
Intensive survey was conducted in different areas of district Ganderbal and Budgam during 2009. Significant variability was recorded with different morphological and physico-chemical properties of pomegranate genotypes. Yield per tree ranged from $(13.80-58.40 \mathrm{Kg})$ and yield efficiency $(0.30-1.58 \mathrm{Kg} / \mathrm{cm} 2)$, fruit length $(5.06-7.30 \mathrm{~cm})$, fruit diameter $(6.16-8.29 \mathrm{~cm})$, fruit weight (120.25-297.50 g), total aril weight (58-194.25 g), weight per aril (0.18-0.29 g). Fruit chemical varied from TSS (12-16\%), juice content (32.85-60.12\%), acidity (0.31-0.46\%), TSS/acid ratio (26.09-45.96), ascorbic acid content (9.23-20.26), reducing sugar content (6.22-9.23\%), total sugars content (7.31-11.23 \%) and anthocyanin content (9.65$19.23 \mathrm{mg} / 100 \mathrm{~g}$ of fruit). The data presented may be helpful in understanding pest resistance among pomegranate.
\end{abstract}

Keywords: Genotypes, Pomegranate, Selection, Survey, Variability

\section{Introduction}

Identifying and preserving genetic diversity is an important factor in any crop improvement programme. Parental varieties identified on the basis of divergence for any breeding programme would be more promising $^{1}$. Grouping or classification of genotypes based on suitable scale is quite imperative to understand the usable variability. Though significance of morphological traits and multivariate analysis for the characterization of pomegranate cultivars has been stressed in some studies ${ }^{2,3}$. Therefore, the objective of the present work was to characterize 16 indigenous pomegranate accessions using pomological and biochemical traits and analyze the contribution of different traits to the overall yield. In order to select superior genotypes for future breeding Programme of pomegranate, the extent of variability with respect to yield and fruit quality is indispensable. Hence, intensive survey of Budgam and Ganderbal districts were carried out for selecting better genotypes having good bearing capacity with high anthocyanin content.

\section{Materials and Methods}

A total of 153 trees were initially labeled based on the interviews with local people and on the data from Directorate of horticulture of Jammu and Kashmir from two districts namely Budgam and Ganderbal. After first observations, many of these trees were excluded because they either showed heavy infestation of anar butterfly or symptoms of cracking or the average fruit weight was lower. Ultimately, 16 of them were selected to be studied further and individual trees were assigned a separate accession numbers. Every accession was evaluated for different yield parameters of tree as per the standard procedures.

${ }^{*}$ Author for correspondence 
Yield efficiency of tree was calculated as per the formula suggested by Westwood and Robert ${ }^{4}$ and expressed in $\mathrm{kg}$ $\mathrm{cm}^{-2}$. Fruits from selected trees were randomly taken for measuring physical attributes like weight, size, rind proportion, juice content by following standard procedures. The total soluble solids were estimated in terms of Brix by using Atago hand refractometer ${ }^{5}$. Acidity, vitamin C, reducing sugar, total sugars and anthocyanin content were determined by following methods of Ranganna ${ }^{6}$. The data was analyzed in R-software as suggested by Gomez and Gomez?

\section{Results and Discussion}

It is evident from Table 1 and 2 that, there is wide variability among yield and fruit physical characters of pomegranate genotypes in Ganderbal and Budgam district accessions. The number of fruits per tree in Ganderbal accessions showed coefficient of variation 31.04 ranged with mean of 180.10 whereas, number of fruits per tree in Budgam accessions ranged showed coefficient of variation 30.37 with mean of 180.20. Yield per tree in Ganderbal accessions also showed variation and ranged from $20 \mathrm{~kg}$ to

Table 1. Yield and fruit physical characteristics of various pomegranate genotypes in Ganderbal district

\begin{tabular}{lccccccccc}
\hline Accession No. & $\begin{array}{c}\text { No. Of } \\
\text { fruits per } \\
\text { tree }\end{array}$ & $\begin{array}{c}\text { Yield/tree } \\
(\mathbf{k g})\end{array}$ & $\begin{array}{c}\text { Yield } \\
\text { efficiency } \\
\left(\mathbf{k g} / \mathbf{c m}^{2}\right)\end{array}$ & $\begin{array}{c}\text { Fruit } \\
\text { length } \\
(\mathbf{c m})\end{array}$ & $\begin{array}{c}\text { Fruit } \\
\text { diameter } \\
(\mathbf{c m})\end{array}$ & $\begin{array}{c}\text { Fruit weight } \\
(\mathbf{g})\end{array}$ & $\begin{array}{c}\text { Total aril } \\
\text { weight }(\mathrm{g})\end{array}$ & $\begin{array}{c}\text { Weight } \\
\text { per aril } \\
(\mathbf{g})\end{array}$ & $\begin{array}{c}\text { Rind } \\
\text { proportion } \\
(\%)\end{array}$ \\
\hline SKAU-Pg-Gb-001 & 83 & 20.00 & 0.30 & 7.14 & 7.97 & 242.50 & 166.00 & 0.21 & 31.54 \\
SKAU-Pg-Gb-002 & 144 & 38.70 & 0.84 & 7.19 & 8.01 & 268.75 & 138.75 & 0.27 & 48.37 \\
SKAU-Pg-Gb-003 & 190 & 44.00 & 0.45 & 6.74 & 7.76 & 236.00 & 137.50 & 0.24 & 41.74 \\
SKAU-Pg-Gb-004 & 245 & 45.00 & 0.62 & 6.85 & 7.18 & 183.75 & 102.00 & 0.19 & 44.49 \\
SKAU-Pg-Gb-005 & 159 & 40.00 & 0.60 & 6.84 & 7.91 & 251.25 & 181.50 & 0.27 & 27.76 \\
SKAU-Pg-Gb-006 & 225 & 43.87 & 0.89 & 6.23 & 7.28 & 195.00 & 137.50 & 0.22 & 29.49 \\
SKAU-Pg-Gb-007 & 144 & 30.52 & 0.52 & 6.69 & 7.48 & 212.50 & 151.75 & 0.22 & 28.58 \\
Mean & 180.1 & 40.14 & .66 & 6.85 & 7.66 & 227.10 & 145.00 & 0.23 & 36.00 \\
+SE & 21.10 & 4.51 & 0.12 & 0.14 & 0.13 & 11.70 & 9.54 & 0.01 & 3.25 \\
Range & $83.00-245.00$ & $20.00-45.00$ & $0.30-0.89$ & $6.23-7.19$ & $7.18-8.01$ & $183.75-268.75$ & $102.00-181.50$ & $0.19-0.27$ & $27.76-48.37$ \\
CV (\%) & 31.04 & 29.74 & 48.48 & 5.55 & 4.45 & 13.65 & 17.40 & 13.04 & 23.89 \\
\hline
\end{tabular}

Table 2. Yield and fruit physical characteristics of various pomegranate genotypes in Budgam district

\begin{tabular}{lccccccccc}
\hline Accession No. & $\begin{array}{c}\text { No. of fruits } \\
\text { per tree }\end{array}$ & $\begin{array}{c}\text { Yield/ tree } \\
(\mathbf{k g})\end{array}$ & $\begin{array}{c}\text { Yield } \\
\text { efficiency } \\
\left(\mathbf{k g} / \mathbf{c m}^{2}\right)\end{array}$ & $\begin{array}{c}\text { Fruit } \\
\text { length } \\
(\mathbf{c m})\end{array}$ & $\begin{array}{c}\text { Fruit } \\
\text { diameter } \\
(\mathbf{c m})\end{array}$ & $\begin{array}{c}\text { Fruit weight } \\
(\mathbf{g})\end{array}$ & $\begin{array}{c}\text { Total aril } \\
\text { weight }(\mathrm{g})\end{array}$ & $\begin{array}{c}\text { Weight per } \\
\text { aril }(\mathrm{g})\end{array}$ & $\begin{array}{c}\text { Rind } \\
\text { proportion } \\
(\%)\end{array}$ \\
\hline SKAU-Pg-Bd-001 & 118 & 20.06 & 0.31 & 6.06 & 6.88 & 166.25 & 58.00 & 0.18 & 65.11 \\
SKAU-Pg-Bd-002 & 94 & 20.70 & 0.65 & 6.92 & 7.20 & 221.25 & 147.75 & 0.28 & 33.22 \\
SKAU-Pg-Bd-003 & 225 & 57.37 & 1.58 & 6.80 & 8.29 & 255.00 & 167.25 & 0.26 & 34.41 \\
SKAU-Pg-Bd-004 & 215 & 45.15 & 1.35 & 6.86 & 7.86 & 210.00 & 138.50 & 0.21 & 34.04 \\
SKAU-Pg-Bd-005 & 115 & 13.8 & 0.6 & 5.54 & 6.16 & 120.25 & 75.50 & 0.25 & 37.21 \\
SKAU-Pg-Bd-006 & 225 & 39.52 & 1.08 & 5.06 & 6.64 & 155.00 & 106.25 & 0.18 & 45.88 \\
SKAU-Pg-Bd-007 & 204 & 40.8 & 0.60 & 6.28 & 7.15 & 188.75 & 120.00 & 0.24 & 36.29 \\
SKAU-Pg-Bd-008 & 196 & 58.40 & 1.18 & 7.30 & 8.22 & 297.50 & 194.25 & 0.29 \\
SKAU-Pg-Bd-009 & 230 & 40.71 & 0.89 & 6.38 & 7.09 & 177.50 & 90.25 & 0.28 & 34.71 \\
Mean & 180.20 & 37.39 & 0.92 & 6.36 & 7.26 & 199.10 & 122.00 & 0.24 \\
+SE & 18.30 & 5.35 & 0.14 & 0.24 & 0.25 & 18.00 & 14.80 & 0.01 \\
Range & $94.00-230.00$ & $13.80-58.40$ & $0.31-1.58$ & $5.06-7.30$ & $6.16-8.29$ & $120.25-297.50$ & $58.00-194.25$ & $0.18-0.29$ & $33.22-65.11$ \\
CV (\%) & 30.47 & 42.95 & 44.56 & 11.32 & 10.19 & 27.07 & 36.31 & 16.66 & 25.80 \\
\hline
\end{tabular}


$45.00 \mathrm{~kg}$ with mean of $40.14 \mathrm{~kg}$ and coefficient of variation 29.74, whereas, yield per tree in Budgam accessions was in the range of $13.80 \mathrm{~kg}$ in accession to $58.40 \mathrm{~kg}$ with mean of 37.39 and coefficient of variation 42.95. Yield efficiency in Ganderbal accessions showed coefficient of variation 48.48, whereas, in Budgam accessions it was 44.56. In Ganderbal accessions fruit length ranged from $6.23 \mathrm{~cm}$ to $7.19 \mathrm{~cm}$ with coefficient of variation 5.55 where as in Budgam di accessions fruit length ranged from 5.06 $\mathrm{cm}$ to $7.30 \mathrm{~cm}$ with coefficient of variation 11.32 . Highest fruit diameter was recorded in Budgam accessions (8.29 $\mathrm{cm}$ ) with variation coefficient of 10.19. Fruit weight in Ganderbal accessions varied from $183.75 \mathrm{~g}$ to $268.75 \mathrm{~g}$ with coefficient of variation 13.65 where as in Budgam accessions fruit weight ranged from $120.25 \mathrm{~g}$ to $297.50 \mathrm{~g}$ with variation coefficient of 27.07. Variation coefficient for total aril weight per fruit in Ganderbal accessions ranged was 17.40 where as in Budgam accessions it was 36.31. Highest coefficient of variation for weight per aril was observed in Budgam accessions (16.66). Rind proportion also showed variability but it was not too wide between two districts. Fruit weight of pomegranate cultivars was found between 150 and $568 \mathrm{~g}^{8}$. Fruit length, fruit diameter and fruit volume recorded by Kanzankay et al. ${ }^{9}$ support our findings. Similar type of variation for yield and fruit physical parameters was recorded in pomegranate by Mir et al. $^{10}$. The wide variation of the pest attack may be due to different genetic make-up and agro climatic conditions.

Table 3 and 4 revealed variation with respect to various fruit chemical characters in Ganderbal and Budgam district respectively. In Ganderbal highest TSS was reported in accession SKAU-Pg-Gb-001 (14\%) with variation coefficient of 6.24 whereas in Budgam district highest TSS was observed in accession SKAU-Pg-Bd-008 (16\%) with variation coefficient of 9.44. In Ganderbal accessions juice content showed variation coefficient of 20.58 whereas in Budgam accessions it exhibited variation coefficient of 21.52. Maximum acidity in Ganderbal accessions was $0.46 \%$ where as in Budgam accessions maximum acidity was $0.40 \%$. Maximum coefficient of variation for TSS/ acid ratio was reported in Ganderbal district accession (17.02). Ascorbic acid content varied from 9.23 to 15.78 $\mathrm{mg} / 100 \mathrm{~g}$ fruit in the genotypes of Ganderbal district where as in Budgam it ranged from 9.53 to $20.2 \mathrm{mg} / 100$ $\mathrm{g}$ fruit. Coefficient of variation for non-reducing sugars was highest in the genotypes of Budgam district 27.65 whereas for total sugars it was highest in the genotypes of Ganderbal district (12.98). Anthocyanin content varied from $9.65 \mathrm{mg} / 100 \mathrm{~g}$ fruit to $18.67 \mathrm{mg} / 100 \mathrm{~g}$ fruit in Ganderbal genotypes with mean of $12.71 \mathrm{mg} / 100 \mathrm{~g}$ fruit and coefficient of variation 22.00, whereas anthocyanin content in Budgam genotypes varied from $9.96 \mathrm{mg} / 100 \mathrm{~g}$ fruit to $19.23 \mathrm{mg} / 100 \mathrm{~g}$ fruit with mean of $14.7 \mathrm{mg} / 100 \mathrm{~g}$ fruit and variation coefficient of 25.00. Variation in fruit chemical parameters may be due to genetic constitution of accessions and may also be affected by agroclimatic conditions, altitude and environment. Ascorbic acid, reducing and total sugar corresponds to the findings of Akbarpaur et al. ${ }^{11}$. Similar variation in anthocyanin content was reported by Mir et al. ${ }^{10}$.

Variation in fruit chemical parameters may reflect the genetic constitution of accessions as influenced by

Table 3. Fruit chemical characteristics of various pomegranate genotypes in Ganderbal district

\begin{tabular}{|c|c|c|c|c|c|c|c|c|}
\hline Accession No. & $\begin{array}{c}\text { Juice } \\
\text { content (\%) }\end{array}$ & $\begin{array}{c}\text { TSS } \\
\text { (oBrix) }\end{array}$ & $\begin{array}{c}\text { Acidity } \\
(\%)\end{array}$ & $\begin{array}{l}\text { TSS/acid } \\
\text { ratio }\end{array}$ & $\begin{array}{c}\text { Ascorbic acid } \\
\text { (mg/100g } \\
\text { fruit })\end{array}$ & $\begin{array}{l}\text { Reducing } \\
\text { sugar (\%) }\end{array}$ & $\begin{array}{c}\text { Anthocyanin } \\
\text { content } \\
(\mathrm{mg} / 100 \mathrm{gm})\end{array}$ & $\begin{array}{c}\text { Total sugar } \\
\text { (\%) }\end{array}$ \\
\hline SKAU-Pg-Gb-001 & 48.62 & 14.00 & 0.32 & 43.75 & 15.78 & 8.83 & 15.22 & 10.23 \\
\hline SKAU-Pg-Gb-002 & 36.50 & 13.37 & 0.38 & 35.18 & 14.97 & 7.12 & 9.65 & 8.78 \\
\hline SKAU-Pg-Gb-003 & 47.28 & 13.50 & 0.35 & 38.57 & 15.69 & 7.80 & 18.67 & 9.23 \\
\hline SKAU-Pg-Gb-004 & 42.60 & 12.00 & 0.41 & 29.27 & 13.83 & 6.22 & 10.43 & 7.31 \\
\hline SKAU-Pg-Gb-005 & 35.88 & 12.25 & 0.37 & 33.11 & 12.62 & 6.34 & 10.32 & 7.68 \\
\hline SKAU-Pg-Gb-006 & 60.21 & 12.63 & 0.34 & 37.15 & 14.83 & 7.25 & 13.77 & 9.22 \\
\hline SKAU-Pg-Gb-007 & 35.67 & 12.00 & 0.46 & 26.09 & 9.23 & 6.49 & 10.88 & 7.43 \\
\hline Mean & 43.82 & 12.82 & 0.38 & 34.73 & 13.85 & 7.15 & 12.71 & 8.55 \\
\hline$+\mathrm{SE}$ & 2.65 & 0.30 & 0.02 & 2.23 & 0.87 & 0.35 & 0.94 & 0.42 \\
\hline Range & $35.67-60.21$ & $12.00-14.00$ & $0.32-0.46$ & $26.09-43.75$ & $9.23-15.78$ & $6.22-8.83$ & 22.00 & $7.31-10.23$ \\
\hline CV (\%) & 20.58 & 6.24 & 13.16 & 17.02 & 16.75 & 13.00 & $9.65-18.67$ & 12.98 \\
\hline
\end{tabular}


Table 4. Fruit chemical characteristics of various pomegranate genotypes in Budgam district

\begin{tabular}{|c|c|c|c|c|c|c|c|c|}
\hline Accession No. & $\begin{array}{c}\text { Juice } \\
\text { content } \\
(\%)\end{array}$ & TSS (oBrix) & Acidity (\%) & $\begin{array}{c}\text { TSS/acid } \\
\text { ratio }\end{array}$ & $\begin{array}{c}\text { Ascorbic acid } \\
\text { (mg/100g } \\
\text { fruit })\end{array}$ & $\begin{array}{l}\text { Reducing } \\
\text { sugar (\%) }\end{array}$ & $\begin{array}{c}\text { Anthocyanin } \\
\text { content } \\
(\mathrm{mg} / 100 \mathrm{gm})\end{array}$ & $\begin{array}{c}\text { Total } \\
\text { sugar }(\%)\end{array}$ \\
\hline SKAU-Pg-Bd-001 & 38.82 & 15.75 & 0.36 & 43.75 & 14.64 & 9.17 & 15.76 & 11.23 \\
\hline SKAU-Pg-Bd-002 & 32.85 & 13.37 & 0.40 & 33.43 & 12.24 & 6.97 & 10.34 & 8.28 \\
\hline SKAU-Pg-Bd-003 & 58.60 & 14.12 & 0.34 & 41.53 & 16.42 & 8.39 & 15.92 & 10.83 \\
\hline SKAU-Pg-Bd-004 & 34.24 & 12.50 & 0.37 & 33.78 & 9.53 & 6.78 & 10.36 & 8.20 \\
\hline SKAU-Pg-Bd-005 & 46.03 & 14.25 & 0.31 & 45.96 & 18.12 & 8.49 & 14.72 & 9.42 \\
\hline SKAU-Pg-Bd-006 & 50.12 & 13.25 & 0.37 & 35.81 & 16.90 & 7.25 & 18.32 & 9.13 \\
\hline SKAU-Pg-Bd-007 & 36.89 & 13.38 & 0.38 & 35.21 & 13.78 & 7.42 & 9.96 & 9.54 \\
\hline SKAU-Pg-Bd-008 & 57.50 & 16.00 & 0.36 & 44.45 & 20.26 & 9.23 & 19.23 & 10.64 \\
\hline SKAU-Pg-Bd-009 & 45.53 & 12.25 & 0.34 & 36.03 & 16.32 & 6.92 & 8.22 & 8.66 \\
\hline Mean & 44.51 & 13.87 & 0.37 & 38.88 & 15.36 & 7.85 & 14.70 & 9.55 \\
\hline$+\mathrm{SE}$ & 2.21 & 0.43 & 0.01 & 1.66 & 1.07 & 0.33 & 0.95 & 0.37 \\
\hline Range & $32.85-58.60$ & $12.25-16.00$ & $0.31-0.40$ & $33.43-45.96$ & $9.53-20.26$ & $6.78-9.23$ & $9.96-19.23$ & $8.20-11.23$ \\
\hline CV (\%) & 21.52 & 9.44 & 8.11 & 12.81 & 20.96 & 12.48 & 25 & 11.73 \\
\hline
\end{tabular}

agroclimatic conditions, altitude and environment. The data presented may also be helpful in understanding pest resistance among pomegranate.

\section{References}

1. Arunachalam G. Genetic distances in plant breeding. Indian J Genet. 1981; 41:226-36.

2. Al-Said FA, Opara LU, Al-Yahyai RA. Physical, chemical and textural quality attributes of pomegranate cultivars (Punicagranaium L.) cultivars in Eastern Mediterranean region of Turkey. AJB. 2009; 7:1294-301.

3. Muradoglu F, Balta FM, Ozrenk K. Pomegranate (Punica granatum L.) genetic resources from Hakkari, Turkey. Resource Journal of Agricultural Biological Science. 2006; 2(6):520-5.

4. Westwood MJN, Roberts AJV. The relationship between trunk cross sectional area and weight of apple. J Am Soc. 1970; 95:23-30.

5. AOAC International. Official Methods of Analysis. 16th ed. Washington, D.C.: Association of Official Analytical Chemists; 1998.
6. Ranganna S. Handbook of Analysis and Quality Control for Fruit and Vegetable Products. 2nd ed. New Delhi: Tata McGraw-Hill; 2001.

7. Gomez KA, Gomez AA. Statistical Procedure for Agriculture Research. 2nd ed. New York: John Wiley and Sons Inc; 1984.

8. Ozkan Y. 2005. Investigation on physical and chemical characteristics of some pomegranate genotypes (Punicagranatum L.) of Tokat province in Turkey. Asian J Chem. 2005; 17:939-42 .

9. Kazankaya A, Gundogdu M, Askin MA, Muradoglu F. Fruit attributes of local pomegranate grown in Pervari. Proceedings of 4th National Horticulture Congress; 2004 Sep 8-12; Antalya, Turkey.

10. Mir MM, Sofi AA, Ahmad I, Beigh MA. Performance of pomegranate cultivars under karewa belts of Kashmir valley. SKUAST Journal of Research. 2007; 9(1):104-8.

11. Akbarpour V, Hemmati K, Sharifani M. 2009. Physical and chemicalpropertiesofpomegranate, fruitinmaturationstage. American Eurasian Journal of Agriculture and Environment Science. 2009; 6(4):411-6. 